\title{
New General Theorems and Explicit Values of the Level 13 Analogue of Rogers-Ramanujan Continued Fraction
}

\author{
Nipen Saikia \\ Department of Mathematics, Rajiv Gandhi University, Rono Hills, Doimukh, Arunachal Pradesh 791112, India \\ Correspondence should be addressed to Nipen Saikia; nipennak@yahoo.com
}

Received 4 September 2014; Accepted 6 November 2014; Published 27 November 2014

Academic Editor: Cheon S. Ryoo

Copyright (C) 2014 Nipen Saikia. This is an open access article distributed under the Creative Commons Attribution License, which permits unrestricted use, distribution, and reproduction in any medium, provided the original work is properly cited.

We prove general theorems for the explicit evaluations of the level 13 analogue of Rogers-Ramanujan continued fraction and find some new explicit values. This work is a sequel to some recent works of S. Cooper and D. Ye.

\section{Introduction}

For $q=e^{2 \pi i z}$ and $\operatorname{Im}(z)>0$, the Dedekind eta function $\eta(z)$ and Ramanujan's function $f(-q)$ are defined by

$$
f(-q):=(q ; q)_{\infty}=: q^{-1 / 24} \eta(z),
$$

where $(a ; q)_{\infty}=\prod_{n=0}^{\infty}\left(1-a q^{n}\right)$. The famous Rogers-Ramanujan continued fraction $\mathscr{R}(q)$ is defined by

$$
\mathscr{R}(q):=q^{1 / 5} \prod_{n=1}^{\infty} \frac{\left(1-q^{5 n-4}\right)\left(1-q^{5 n-1}\right)}{\left(1-q^{5 n-3}\right)\left(1-q^{5 n-2}\right)} .
$$

This continued fraction was introduced by Rogers [1] in 1894 and rediscovered by Ramanujan in approximately 1912. In his notebooks [2], lost notebook [3], and his first two letters to Hardy [4], Ramanujan recorded several explicit values of $\mathscr{R}(q)$. These values are first proved by Watson $[5,6]$ and Ramanathan [7]. For further references on explicit evaluations of $\mathscr{R}(q)$ see [8-14].

The Rogers-Ramanujan continued fraction $\mathscr{R}(q)$ is closely related to the function $f(-q)$ by the following two beautiful relations:

$$
\begin{aligned}
& \frac{1}{\mathscr{R}(q)}-1-\mathscr{R}(q)=\frac{f\left(-q^{1 / 5}\right)}{q^{1 / 5} f\left(-q^{5}\right)}, \\
& \frac{1}{\mathscr{R}^{5}(q)}-11-\mathscr{R}^{5}(q)=\frac{f^{6}(-q)}{q f^{6}\left(-q^{5}\right)},
\end{aligned}
$$

which are stated by Ramanujan [15, page 267, (11.6)] and first proved by Watson [5].

In his second notebook [2, 15, Chapter 20, Entry 8(i)] Ramanujan stated an interesting analogue of $\mathscr{R}(q)$. If

$$
\begin{aligned}
R(q):=q \prod_{n=1}^{\infty} & \left(\left(1-q^{13 n-12}\right)\left(1-q^{13 n-10}\right)\right. \\
& \times\left(1-q^{13 n-9}\right)\left(1-q^{13 n-4}\right) \\
& \left.\times\left(1-q^{13 n-3}\right)\left(1-q^{13 n-1}\right)\right) \\
\times & \left(\left(1-q^{13 n-11}\right)\left(1-q^{13 n-8}\right)\right. \\
& \times\left(1-q^{13 n-7}\right)\left(1-q^{13 n-6}\right) \\
& \left.\times\left(1-q^{13 n-5}\right)\left(1-q^{13 n-2}\right)\right)^{-1},
\end{aligned}
$$

then

$$
\frac{1}{R(q)}-3-R(q)=\frac{f^{2}(-q)}{q f^{2}\left(-q^{13}\right)}
$$

Proofs of (5) can be found in $[16,17]$. The function $R(q)$ is studied by Cooper and Ye [18, 19]. In [18] Cooper and Ye evaluated or indicated the explicit values of $R\left(e^{-2 \pi \sqrt{n / 13}}\right)$ and $R\left(-e^{-\pi \sqrt{n / 13}}\right)$ for $n=1,2,3,5,7,9,13,15,31,55,69,129,231$, and 255 by using the methods of reciprocity formulas, $P-Q$ modular equations, Ramanujan-Weber class invariant, and 
Kronecker's limit formula and in terms of the functions $F(q)$ and $T(q)[18$, page $94,(1.7)$ and (1.8)] which are defined by

$$
\begin{aligned}
F(q) & :=\frac{1}{R(q)}-3-R(q)=\frac{f^{2}(-q)}{q f^{2}\left(-q^{13}\right)}, \\
T(q) & :=\frac{F(q)}{F^{2}(q)+6 F(q)+13} \\
& =\frac{R(q)\left(1-3 R(q)-R^{2}(q)\right)}{\left(1+R^{2}(q)\right)^{2}} .
\end{aligned}
$$

From (6) and (7), it is clear that if we know $F(q)$ or $T(q)$ for any particular value of $q$, then $R(q)$ can be determined by solving the corresponding quadratic equations.

Cooper and Ye [18, page 104, Theorem 4.1] further established that

$$
\begin{aligned}
& \frac{F\left(q^{2}\right)}{V(q)}-\frac{13 V(q)}{F\left(q^{2}\right)} \\
& \quad=-F(-q) V(q)+\frac{13}{F(-q) V(q)} \\
& \quad=\left(V(q)-\frac{1}{V(q)}\right)^{3}+7\left(V(q)-\frac{1}{V(q)}\right),
\end{aligned}
$$

where

$$
V(q)=\frac{\chi\left(q^{13}\right)}{q^{1 / 2} \chi(q)}
$$

and $\chi(q)=\left(-q ; q^{2}\right)_{\infty}$. They also indicated that if we know $V(q)$ for any particular $q$, then $F\left(q^{2}\right)$ and $F(-q)$ can be determined by appealing to (8) and therefrom $R\left(q^{2}\right)$ and $R(-q)$ can be determined at the same $q$. For this they evaluated $V\left(e^{-\pi \sqrt{n / 13}}\right)$ for $n=5,9,13,69$, and 129 .

In this paper, we prove some general theorems for the explicit evaluations $R(q)$ and $R(-q)$ by parameterizations of Dedekind eta function and find some old and new explicit values. In Section 2, we record some preliminary results which will be used in the subsequent sections. In Section 3, we prove general theorems for the explicit evaluations of $R( \pm q)$ and evaluate some old and new explicit values. In Section 4, we find some new explicit values of $V(q)$ by parametrization. Finally, in Section 5, we consider the function $F(q)$.

\section{Preliminaries}

Lemma 1 (see [15, page 43, Entry 27(iii), (iv), (vi)]). If $\alpha$ and $\beta$ are such that the modulus of each exponential argument below is less than 1 and $\alpha \beta=\pi^{2}$, then

$$
\begin{aligned}
e^{-\alpha / 12 \sqrt[4]{\alpha}} f\left(-e^{-2 \alpha}\right) & =e^{-\beta / 12} \sqrt[4]{\beta} f\left(-e^{-2 \beta}\right), \\
e^{-\alpha / 24} \sqrt[4]{\alpha} f\left(e^{-\alpha}\right) & =e^{-\beta / 24} \sqrt[4]{\beta} f\left(e^{-\beta}\right) \\
e^{\alpha / 24} \chi\left(e^{-\alpha}\right) & =e^{\beta / 24} \chi\left(e^{-\beta}\right) .
\end{aligned}
$$

Lemma 2 (see [20, page 211, Entry 57]). If $P=f(-q) /$ $q^{1 / 2} f\left(-q^{13}\right)$ and $Q=f\left(-q^{2}\right) / q f\left(-q^{26}\right)$, then

$$
P Q+\frac{13}{P Q}=\left(\frac{P}{Q}\right)^{3}+\left(\frac{Q}{P}\right)^{3}-4\left(\frac{P}{Q}+\frac{Q}{P}\right) .
$$

Lemma 3 (see [20, page 237, Entry 72]). If $P=f(-q) /$ $q^{1 / 2} f\left(-q^{13}\right)$ and $Q=f\left(-q^{3}\right) / q^{3 / 2} f\left(-q^{39}\right)$, then

$$
P Q+\frac{13}{P Q}=\left(\frac{P}{Q}\right)^{2}+\left(\frac{Q}{P}\right)^{2}-3\left(\frac{P}{Q}+\frac{Q}{P}\right)-3
$$

\section{General Theorems for Explicit Evaluations of $R( \pm q)$}

In this section we prove general theorems for the explicit evaluations of $R( \pm q)$ and find some explicit values.

Theorem 4. One has the following.

(i) For $q=e^{-2 \pi \sqrt{n / 13}}$, let

$$
s_{n}=\frac{f(-q)}{(13)^{1 / 4} q^{1 / 2} f\left(-q^{13}\right)} .
$$

Then

$$
\frac{1}{R\left(e^{-2 \pi \sqrt{n / 13}}\right)}-3-R\left(e^{-2 \pi \sqrt{n / 13}}\right)=\sqrt{13} s_{n}^{2} .
$$

(ii) For $q=e^{-\pi \sqrt{n / 13}}$, let

$$
t_{n}=\frac{f(q)}{(13)^{1 / 4} q^{1 / 2} f\left(q^{13}\right)} .
$$

Then

$$
R\left(-e^{-\pi \sqrt{n / 13}}\right)+3-\frac{1}{R\left(-e^{-\pi \sqrt{n / 13}}\right)}=\sqrt{13} t_{n}^{2} .
$$

Proof. We set $q:=e^{-2 \pi \sqrt{n / 13}}$ in (5) and use the definition of $s_{n}$ to arrive at (i). To prove (ii) we replace $q$ by $-q$ in (5), set $q=e^{-\pi \sqrt{n / 13}}$, and use the definition of $t_{n}$.

Theorem 5. If $s_{n}$ and $t_{n}$ are as defined in Theorem 4, then

$$
s_{1 / n}=\frac{1}{s_{n}}, \quad t_{1 / n}=\frac{1}{t_{n}} .
$$

Proof. We use the definitions of $s_{n}$ and $t_{n}$ and use (10) and (11), respectively, to complete the proof.

Corollary 6. If $s_{n}$ and $t_{n}$ are as defined in Theorem 4, then
(i) $1 / R\left(e^{-2 \pi / \sqrt{13 n}}\right)-3-R\left(e^{-2 \pi / \sqrt{13 n}}\right)=\sqrt{13} / s_{n}^{2}$,
(ii) $R\left(-e^{-\pi / \sqrt{13 n}}\right)+3-1 / R\left(-e^{-\pi / \sqrt{13 n}}\right)=\sqrt{13} / t_{n}^{2}$. 
Proof. We replace $n$ by $1 / n$ in Theorem 4(i) and (ii) and employ Theorem 5 to arrive at (i) and (ii), respectively.

From Theorem 4(i) and Corollary 6(i), it is clear that if we know the explicit values of the parameter $s_{n}$, then explicit values of $R\left(e^{-2 \pi \sqrt{n / 13}}\right)$ and $R\left(e^{-2 \pi / \sqrt{13 n}}\right)$ can be evaluated, respectively. Similarly, if we know the explicit values of the parameter $t_{n}$, then explicit values of $R\left(-e^{-\pi \sqrt{n / 13}}\right)$ and $R\left(-e^{-\pi / \sqrt{13 n}}\right)$ can be determined from Theorem 4(ii) and Corollary 6(ii), respectively. $t_{n}$.

Next we find some explicit values of the parameters $s_{n}$ and

Corollary 7. One has

$$
s_{1}=1, \quad t_{1}=1
$$

Proof. We set $n=1$ in Theorem 5 to complete the proof.

Remark 8. Setting $n=1$ in Theorem 4(i), employing the values $s_{1}=1$, and solving the resulting equation, we evaluate

$$
R\left(e^{-2 \pi / \sqrt{13}}\right)=\frac{(-3-\sqrt{13}+\sqrt{26+6 \sqrt{13}})}{2} .
$$

Similarly, setting $n=1$ in Theorem 4(ii), employing the values $t_{1}=1$, and solving the resulting equation, we evaluate

$$
R\left(-e^{-\pi / \sqrt{13}}\right)=\frac{(-3+\sqrt{13}-\sqrt{26+6 \sqrt{13}})}{2} .
$$

The values $R\left(e^{-2 \pi / \sqrt{13}}\right)$ and $R\left(-e^{-\pi / \sqrt{13}}\right)$ are also evaluated by Cooper and Ye [18] by using reciprocity formulas.

Theorem 9. One has

$$
\sqrt{13}\left(s_{n} s_{4 n}+\frac{1}{s_{n} s_{4 n}}\right)=\left(\frac{s_{n}}{s_{4 n}}\right)^{3}+\left(\frac{s_{4 n}}{s_{n}}\right)^{3}-4\left(\frac{s_{n}}{s_{4 n}}+\frac{s_{4 n}}{s_{n}}\right) .
$$

Proof. We use the definition of $s_{n}$ in Lemma 2 to complete the proof.

Corollary 10. One has

$$
\text { (i) } \begin{aligned}
s_{4}= & \frac{(\sqrt{3+\sqrt{13}}+\sqrt{7+\sqrt{17}})}{2} \text { (ii) } s_{2}^{2}=\frac{1}{6}\left[(-6 \sqrt{6}+27 \sqrt{17})^{1 / 3}+(6 \sqrt{6}+27 \sqrt{17})^{1 / 3}\right] \\
+ & \frac{1}{6}\left[\left(-36+\left((-6 \sqrt{6}+27 \sqrt{17})^{1 / 3}\right.\right.\right. \\
& \left.\left.\left.+(6 \sqrt{6}+27 \sqrt{17})^{1 / 3}\right)^{2}\right)^{1 / 2}\right] .
\end{aligned}
$$

Proof. Setting $n=1$ in Theorem 9 and noting $s_{1}=1$ from Corollary 7 , we obtain

$$
(4+\sqrt{13})\left(s_{4}+\frac{1}{s_{4}}\right)-\left(s_{4}^{3}+\frac{1}{s_{4}^{3}}\right)=0 .
$$

Set

$$
s_{4}+\frac{1}{s_{4}}=y
$$

Employing (26) in (25) and simplifying, we obtain

$$
y=(7+\sqrt{13})^{1 / 2}
$$

Employing (27) in (26), solving the resulting equation, and noting $s_{4}>1$, we arrive at (i).

To prove (ii), we set $n=1 / 2$ in Theorem 9 and noting $s_{1 / n}=1 / s_{n}$ from Theorem 5 , we obtain

$$
s_{2}^{6}+\frac{1}{s_{2}^{6}}-4\left(s_{2}^{2}+\frac{1}{s_{2}^{2}}\right)-2 \sqrt{13}=0 .
$$

Solving (28) and choosing the appropriate root, we complete the proof.

Remark 11. Employing the value of $s_{4}$ in Theorem 4(i) and Corollary 6(i) and solving the resulting equations, we evaluate new explicit values

$$
\begin{aligned}
& R\left(e^{-4 \pi / \sqrt{13}}\right) \\
& =4(19+5 \sqrt{13}+\sqrt{442+130 \sqrt{13}} \\
& \left.+\sqrt{16+(19+5 \sqrt{13}+\sqrt{442+130 \sqrt{13}})^{2}}\right)^{-1},
\end{aligned}
$$

$$
\begin{aligned}
& R\left(e^{-\pi / \sqrt{13}}\right) \\
& =(-15-5 \sqrt{13}-3 \sqrt{34+10 \sqrt{13}} \\
& +(1144+320 \sqrt{13}+130 \sqrt{34+10 \sqrt{13}} \\
& \left.\quad+38 \sqrt{442+130 \sqrt{13}})^{1 / 2}\right) \\
& \quad \times(2(5+\sqrt{13}+\sqrt{34+10 \sqrt{13}}))^{-1},
\end{aligned}
$$

respectively. Similarly, by employing the value of $s_{2}$ in Theorem 4(i) and Corollary 6(i) and solving the resulting 
equations we can evaluate explicit values of $R\left(e^{-2 \pi \sqrt{2 / 13}}\right)$ and $R\left(e^{-\pi \sqrt{2 / 13}}\right)$, respectively. The value $R\left(e^{-2 \pi \sqrt{2 / 13}}\right)$ is also indicated by Cooper and Ye [18].

Theorem 12. One has

$$
\left(s_{n} s_{9 n}+\frac{1}{s_{n} s_{9 n}}\right)=\left(\frac{s_{n}}{s_{9 n}}\right)^{2}+\left(\frac{s_{9 n}}{s_{n}}\right)^{2}-3\left(\frac{s_{n}}{s_{9 n}}+\frac{s_{9 n}}{s_{n}}\right)-3 .
$$

Proof. We employ the definition of $s_{n}$ in Lemma 3 to complete the proof.

Theorem 13. One has

$$
\begin{aligned}
\text { (i) } s_{9}= & (3+\sqrt{13}+\sqrt{6(7+\sqrt{13})} \\
& \left.+\sqrt{-6+(3+\sqrt{13}+\sqrt{6(7+\sqrt{13})})^{2}}\right) \\
& \times(4)^{-1}, \\
\text { (ii) } s_{3}^{2}= & \frac{(7+\sqrt{13}+\sqrt{46+14 \sqrt{13}})}{4} .
\end{aligned}
$$

Proof. Setting $n=1$ in Theorem 12 and using the result $s_{1}=1$, we obtain

$$
\sqrt{13}\left(s_{9}+\frac{1}{s_{9}}\right)=s_{9}^{2}+\frac{1}{s_{9}^{2}}-3\left(s_{9}+\frac{1}{s_{9}}\right)-3 .
$$

Solving (33) and choosing the appropriate root, we arrive at (i).

Again, setting $n=1 / 3$ in Theorem 12 and simplifying using Theorem 5 , we obtain

$$
2 \sqrt{13}=s_{3}^{4}+\frac{1}{s_{3}^{4}}-3\left(s_{3}^{2}+\frac{1}{s_{3}^{2}}\right)-3 .
$$

Solving (34) and choosing the appropriate root, we complete the proof of (ii).

Remark 14. Employing the value of $s_{9}$ in Theorem 4(i) and Corollary 6(i) and solving the resulting equations we evaluate $R\left(e^{-6 \pi / \sqrt{13}}\right)$ and $R\left(e^{-2 \pi / 3 \sqrt{13}}\right)$, respectively. Similarly, by employing the value of $s_{3}$ in Theorem 4(i) and Corollary 6(i) and solving the resulting equations, we determine the explicit values of $R\left(e^{-2 \pi \sqrt{3 / 13}}\right)$ and $R\left(e^{-2 \pi / \sqrt{39}}\right)$, respectively.

Theorem 15. One has

$$
\begin{aligned}
\sqrt{13}\left(t_{n} t_{9 n}+\frac{1}{t_{n} t_{9 n}}\right)= & \left(\frac{t_{n}}{t_{9 n}}\right)^{2}+\left(\frac{t_{9 n}}{t_{n}}\right)^{2} \\
& +3\left(\frac{t_{n}}{t_{9 n}}+\frac{t_{9 n}}{t_{n}}\right)-3 .
\end{aligned}
$$

Proof. We replace $q$ by $-q$ in Lemma 3 and employ the definition of $t_{n}$.

Theorem 16. One has

$$
\text { (i) } \begin{aligned}
t_{9}= & -3+\sqrt{13}+\sqrt{42-6 \sqrt{13}} \\
& \left.+\sqrt{-16+(-3+\sqrt{13}+\sqrt{42-6 \sqrt{13}})^{2}}\right) \\
& \times(4)^{-1},
\end{aligned}
$$

(ii) $t_{3}^{2}=\frac{(1+\sqrt{13}+\sqrt{-2+2 \sqrt{13}})}{4}$.

Proof. We set $n=1$ in Theorem 15, employ $t_{1}=1$ from Corollary 7 , and solve the resulting equation to arrive at (i). To prove (ii) we set $n=1 / 3$, employ the result $t_{1 / n}=1 / t_{n}$ from Theorem 5 , and solve the resulting equation. We complete the proof.

Remark 17. Employing the value of $t_{9}$ in Theorem 4(ii) and Corollary 6(ii) and solving the resulting equations, we can evaluate the explicit values of $R\left(-e^{-3 \pi / \sqrt{13}}\right)$ and $R\left(-e^{-\pi / 3 \sqrt{13}}\right)$, respectively. Similarly, by employing the value of $t_{3}$ in Theorem 4(ii) and Corollary 6(ii) and solving the resulting equations, we can calculate the values of $R\left(-e^{-\pi \sqrt{3 / 13}}\right)$ and $R\left(-e^{-\pi / \sqrt{39}}\right)$, respectively.

\section{Explicit Evaluations of $V(q)$}

In this section we evaluate explicit values of the function $V(q)$ defined in (9) by parametrization method. Cooper and Ye [18] used Ramanujan-Weber class invariants to evaluate $V(q)$.

Theorem 18. For $q=e^{-\pi \sqrt{n / 13}}$, let

$$
I_{13, n}=\frac{\chi(q)}{q^{-1 / 2} \chi\left(q^{13}\right)} .
$$

Then

$$
V\left(e^{-\pi \sqrt{n / 13}}\right)=\frac{1}{I_{13, n}} .
$$

Proof. We set $q=e^{-\pi \sqrt{n / 13}}$ in (9) and use the definition of $I_{13, n}$ to complete the proof.

Theorem 19. If $I_{13, n}$ is as defined in Theorem 18, then

(i) $I_{13,1 / n}=1 / I_{13, n}$,

(ii) $V\left(e^{-\pi \sqrt{13 n}}\right)=I_{13, n}$.

Proof. (i) follows easily from the definition of $I_{13, n}$ and (12) or see [21, Theorem 16(ii)]. To prove (ii) we replace $n$ by $1 / n$ in Theorem 18 and use part (i). 
From Theorems 18 and 19(ii) it is clear that if we know the explicit values of $I_{13, n}$ then explicit values of $V\left(e^{-\pi \sqrt{n / 13}}\right)$ and $V\left(e^{-\pi \sqrt{13 n}}\right)$ can be determined. Many explicit values of the parameter $I_{13, n}$ are evaluated in [21]. In the next theorem we find some explicit values of $V(q)$ by employing the explicit values of $I_{13, n}$ from [21, Corollaries 32 and 34] in Theorem 18.

Theorem 20. One has the following:

(i) $I_{13,1}=V\left(e^{-\pi / \sqrt{13}}\right)=1$,

(ii) $I_{13,1 / 3}=V\left(e^{-\pi \sqrt{3 / 13}}\right)=(1+\sqrt{13}+\sqrt{-2+2 \sqrt{13}}) / 4$,

(iii) $I_{13,3}=V\left(e^{-\pi / \sqrt{39}}\right)=(1+\sqrt{13}-\sqrt{-2+2 \sqrt{13}}) / 4$,

(iv) $I_{13,1 / 9}=V\left(e^{-3 \pi / \sqrt{13}}\right)=(2+\sqrt{3}+\sqrt{3+4 \sqrt{3}}) / 2$,

(v) $I_{13,9}=V\left(e^{-\pi / \sqrt{117}}\right)=(2+\sqrt{3}-\sqrt{3+4 \sqrt{3}}) / 2$,

(vi) $I_{13,1 / 5}=V\left(e^{-\pi \sqrt{5 / 13}}\right)=(\sqrt{3+\sqrt{65}}+\sqrt{58+6 \sqrt{65}}) / 2$,

(vii) $I_{13,5}=V\left(e^{-\pi / \sqrt{65}}\right)=(\sqrt{3+\sqrt{65}}-\sqrt{58+6 \sqrt{65}}) / 2$,

(viii) $I_{13,1 / 25}=V\left(e^{-5 \pi / \sqrt{13}}\right)=\left(C+\sqrt{-36+C^{2}}\right) / 6$,

(ix) $I_{13,25}=V\left(e^{-\pi / \sqrt{325}}\right)=\left(C-\sqrt{-36+C^{2}}\right) / 6$,

where $C=6+(1080-15 \sqrt{39})^{1 / 3}+(1080+15 \sqrt{39})^{1 / 3}$.

Remark 21. (i) The values $V\left(e^{-3 \pi / \sqrt{13}}\right)$ and $V\left(e^{-\pi \sqrt{5 / 13}}\right)$ are also evaluated in [18] using Ramanujan's class invariants $G_{n}$. The remaining values of $V(q)$ in Theorem 20 are new.

(ii) The values $V\left(e^{-5 \pi / \sqrt{13}}\right)$ and $V\left(e^{-\pi / \sqrt{325}}\right)$ can be used to evaluate new explicit values $F\left(e^{-10 \pi / \sqrt{13}}\right)$ (or $R\left(e^{-10 \pi / \sqrt{13}}\right)$ ) and $F\left(e^{-2 \pi / \sqrt{325}}\right)$ (or $R\left(e^{-2 \pi / \sqrt{325}}\right)$ ), respectively, by appealing to (6) and (8). Similarly, the explicit values $V\left(e^{-5 \pi / \sqrt{13}}\right)$ and $V\left(e^{-\pi / \sqrt{325}}\right)$ can be used to evaluate new explicit values $-F\left(-e^{-5 \pi / \sqrt{13}}\right)$ (or $R\left(-e^{-5 \pi / \sqrt{13}}\right)$ ) and $-F\left(-e^{-\pi / \sqrt{325}}\right.$ ) (or $R\left(-e^{-\pi / \sqrt{325}}\right)$ ), respectively, by appealing to (6) and (8).

Theorem 22. One has the following:

(i) $V\left(e^{-\pi \alpha}\right) V\left(e^{-\pi \beta}\right)=1$, for $\alpha \beta=1 / 13$,

(ii) $V\left(e^{-\pi \sqrt{n / 13}}\right) V\left(e^{-\pi / \sqrt{13 n}}\right)=1$.

Proof. (i) follows from the definition of $V(q)$ and (12). (ii) follows from Theorems 18 and 19(ii) or from part (i) with $\alpha=\sqrt{n / 13}$ and $\beta=1 / \sqrt{13 n}$.

Remark 23. Theorem 22(ii) implies that if we know explicit value of any one of $V\left(e^{-\pi \sqrt{n / 13}}\right)$ and $V\left(e^{-\pi / \sqrt{13 n}}\right)$, then others can be determined. Cooper and Ye [18, pages 107108, Theorems 4.4, 4.5, and 4.6] evaluated explicit values of $V\left(e^{-\pi \sqrt{n / 13}}\right)$ for $n=13,69$, and 129 by using Weber-Ramanujan class invariants. Employing these values of $V\left(e^{-\pi \sqrt{n / 13}}\right)$ in Theorem 22(ii) we can evaluate $V\left(e^{-\pi / \sqrt{n}}\right)$ for $n=169,897$, and 1677, respectively. For example, setting $n=129$ in Theorem 22(ii) and employing the value

$$
\begin{aligned}
V\left(e^{-\pi \sqrt{129 / 13}}\right)= & \frac{1}{4}(\sqrt{355+54 \sqrt{43}}+\sqrt{351+54 \sqrt{43}}) \\
& \times(\sqrt{17+2 \sqrt{43}}+\sqrt{13+2 \sqrt{43}}),
\end{aligned}
$$

from [18, page 108, Theorem 4.6], we evaluate

$$
\begin{aligned}
V\left(e^{-\pi / \sqrt{1677}}\right)= & \frac{1}{4}(\sqrt{355+54 \sqrt{43}}-\sqrt{351+54 \sqrt{43}}) \\
& \times(\sqrt{17+2 \sqrt{43}}-\sqrt{13+2 \sqrt{43}}) .
\end{aligned}
$$

The explicit values $V\left(e^{-\pi / \sqrt{n}}\right)$ for $n=169,897$, and 1677 can be used to calculate new explicit values $F\left(e^{-2 \pi / 13}\right.$ ) (or $\left.R\left(e^{-2 \pi / 13}\right)\right), F\left(e^{-2 \pi / \sqrt{897}}\right)$ (or $\left.R\left(e^{-2 \pi / \sqrt{897}}\right)\right)$, and $F\left(e^{-2 \pi / \sqrt{1677}}\right)$ (or $R\left(e^{-2 \pi / \sqrt{1677}}\right)$ ), respectively, by appealing to (6) and (8). Similarly, we can use these values of $V\left(e^{-\pi / \sqrt{n}}\right)$ to evaluate new explicit values $F\left(-e^{-2 \pi / 13}\right)$ (or $\left.R\left(-e^{-2 \pi / 13}\right)\right),\left(-e^{-2 \pi / \sqrt{897}}\right)$ (or $R\left(-e^{-2 \pi / \sqrt{897}}\right)$ ), and $F\left(-e^{-2 \pi / \sqrt{1677}}\right)$ (or $R\left(-e^{-2 \pi / \sqrt{1677}}\right)$ ), respectively, by appealing to (6) and (8).

\section{Explicit Evaluations of $F(q)$}

Theorem 24. If $t_{n}$ is as defined in Theorem 4, then

$$
\begin{aligned}
& \text { (i) }-F\left(-e^{-\pi \sqrt{n / 13}}\right)=\sqrt{13} t_{n}^{2}, \\
& \text { (ii) }-F\left(-e^{-\pi / \sqrt{13 n}}\right)=\sqrt{13} / t_{n}^{2} .
\end{aligned}
$$

Proof. Replacing $q$ by $-q$ in (6) and employing the definition of $t_{n}$ we arrive at (i). To prove (ii) we replace $n$ by $1 / n$ in part (i) and use the result $t_{1 / n}=1 / t_{n}$ from Theorem 5 .

Theorem 25. One has the following:

(i) $F\left(-e^{-\pi \alpha}\right) F\left(-e^{-\pi \beta}\right)=13$, for $\alpha \beta=1 / 13$,

(ii) $F\left(-e^{-\pi \sqrt{n / 13}}\right) F\left(-e^{-\pi / \sqrt{13 n}}\right)=13$.

Proof. (i) follows from the definition of $F(q)$ from (6) with $q$ replaced by $-q$ and (11). (ii) follows from Theorem 24(i) and (ii) or from part (i) with $\alpha=\sqrt{n / 13}$ and $\beta=1 / \sqrt{13 n}$.

Remark 26. Theorem 25(ii) implies that if we know explicit value of any one of $F\left(-e^{-\pi \sqrt{n / 13}}\right)$ and $F\left(-e^{-\pi / \sqrt{13 n}}\right)$, then others can be determined. Using Kronecker's limit formula Cooper and Ye [18, page 109, Theorem 5.2] evaluated $F\left(-e^{-\pi \sqrt{n / 13}}\right)$ for $n=15,31,55,231$, and 255. Employing these values of $F\left(-e^{-\pi \sqrt{n / 13}}\right)$ in Theorem 25(ii) we can evaluate explicit value of $F\left(-e^{-\pi / \sqrt{n}}\right)$ for $n=195,403,715,3003$, and 
3315, respectively. For example, setting $n=31$ in Theorem 25(ii) and employing the value

$$
F\left(-e^{-\pi \sqrt{31 / 13}}\right)=-\sqrt{13}\left(\frac{\sqrt{13}+3}{2}\right)^{3},
$$

we evaluate

$$
F\left(-e^{-\pi / \sqrt{403}}\right)=-\left(\frac{\sqrt{13}-3}{2}\right)^{3} .
$$

The values $F\left(-e^{-\pi / \sqrt{n}}\right)$ for $n=195,403,715,3003$, and 3315 can be used to find new explicit values $R\left(-e^{-\pi / \sqrt{195}}\right), R\left(-e^{-\pi / \sqrt{403}}\right)$, $R\left(-e^{-\pi / \sqrt{715}}\right), R\left(-e^{-\pi / \sqrt{3003}}\right)$, and $R\left(-e^{-\pi / \sqrt{3315}}\right)$, respectively.

Theorem 27. One has the following:
(i) $F\left(e^{-2 \pi \sqrt{n / 13}}\right)=\sqrt{13} s_{n}^{2}$,
(ii) $F\left(e^{-2 \pi / \sqrt{13 n}}\right)=\sqrt{13} / s_{n}^{2}$.

Proof. We employ the definition of $s_{n}$ in (6) to arrive at (i). To prove (ii) we replace $n$ by $1 / n$ in part (i) and use the result $s_{1 / n}=1 / s_{n}$ from Theorem 5 .

Theorem 28. One has the following:
(i) $F\left(e^{-2 \pi \alpha}\right) F\left(e^{-2 \pi \beta}\right)=13$, for $\alpha \beta=1 / 13$,
(ii) $F\left(e^{-2 \pi \sqrt{n / 13}}\right) F\left(e^{-2 \pi / \sqrt{13 n}}\right)=13$.

Proof. (i) follows from the definition of $F(q)$ from (6) and (10). (ii) follows from Theorem 27(i) and (ii) or from part (i) with $\alpha=\sqrt{n / 13}$ and $\beta=1 / \sqrt{13 n}$.

Corollary 29. One has the following:
(i) $F\left(e^{-2 \pi \alpha}\right) F\left(e^{-2 \pi \beta}\right)=F\left(-e^{-\pi \alpha}\right) F\left(-e^{-\pi \beta}\right)$, for $\alpha \beta=1 / 13$,
(ii) $F\left(e^{-2 \pi \sqrt{n / 13}}\right) F\left(e^{-2 \pi / \sqrt{13 n}}\right)=F\left(-e^{-\pi \sqrt{n / 13}}\right) F\left(-e^{-\pi / \sqrt{13 n}}\right)$.

Proof. (i) follows from part (i) of Theorems 25 and 28. To prove (ii) we use part (ii) of Theorems 25 and 28.

\section{Conflict of Interests}

The author declares that there is no conflict of interests regarding the publication of this paper.

\section{References}

[1] L. J. Rogers, "Second memoir on the expansion of certain infinite products," Proceedings of the London Mathematical Society, vol. 25, no. 1, pp. 318-343, 1894.

[2] S. Ramanujan, Notebooks, vol. 1-2, Tata Institute of Fundamental Research, Bombay, India, 1957.

[3] S. Ramanujan, The Lost Notebook and Other Unpublished Papers, Narosa, New Delhi, India, 1988.

[4] B. C. Berndt and R. A. Rankin, Ramanujan: Letters and Commentary, American Mathematical Society, Providence, RI, USA, 1995, jointly published by the London Mathematical Society, London, UK, 1995.
[5] G. N. Watson, “Theorems stated by Ramanujan (VII): theorems on continued fractions," Journal of the London Mathematical Society, vol. 4, pp. 39-48, 1929.

[6] G. N. Watson, "Theorems stated by Ramanujan (IX): two continued fractions," Journal of the London Mathematical Society, vol. 4, pp. 231-237, 1929.

[7] K. G. Ramanathan, “On Ramanujan's continued fraction," Acta Arithmetica, vol. 43, no. 3, pp. 209-226, 1984.

[8] N. D. Baruah and N. Saikia, "Some new explicit values of Ramanujan's continued fractions," Indian Journal of Mathematics, vol. 46, no. 2-3, pp. 197-222, 2004.

[9] B. C. Berndt and H. H. Chan, "Some values for the RogersRamanujan continued fraction," Canadian Journal of Mathematics, vol. 47, no. 5, pp. 897-914, 1995.

[10] B. C. Berndt, H. H. Chan, and L.-C. Zhang, "Explicit evaluations of the Rogers-Ramanujan continued fraction," Journal für die Reine und Angewandte Mathematik, vol. 480, pp. 141-159, 1996.

[11] S.-Y. Kang, "Ramanujan's formulas for the explicit evaluation of the Rogers-Ramanujan continued fraction and thetafunctions," Acta Arithmetica, vol. 90, no. 1, pp. 49-68, 1999.

[12] S.-Y. Kang, "Some theorems on the Rogers-Ramanujan continued fraction and associated theta function identities in Ramanujans Lost Notebook," The Ramanujan Journal, vol. 3, pp. 91-111, 1999.

[13] N. Saikia, "Some new explicit values of the parameters $s_{n}$ and $t_{n}$ connected with Rogers-Ramanujan continued fraction and applications," Afrika Matematika, vol. 25, no. 4, pp. 961-973, 2014.

[14] J. Yi, "Evaluations of the Rogers-Ramanujan continued fraction $R(q)$ by modular equations," Acta Arithmetica, vol. 97, no. 2, pp. 103-127, 2001.

[15] B. C. Berndt, Ramanujan's Notebooks. Part III, Springer, New York, NY, USA, 1991.

[16] R. J. Evans, "Theta function identities," Journal of Mathematical Analysis and Applications, vol. 147, no. 1, pp. 97-121, 1990.

[17] T. Horie and N. Kanou, "Certain modular functions similar to the Dedekind eta function," Abhandlungen aus dem Mathematischen Seminar der Universität Hamburg, vol. 72, pp. 89-117, 2002.

[18] S. Cooper and D. Ye, "Explicit evaluations of a level 13 analogue of the Rogers-Ramanujan continued fraction," Journal of Number Theory, vol. 139, pp. 91-111, 2014.

[19] S. Cooper and D. Ye, "The Rogers-Ramanujan continued fraction and its level 13 analogue," Journal of Approximation Theory, 2014.

[20] B. C. Berndt, Ramanujan's Notebooks, Part IV, Springer, New York, NY, USA, 1994.

[21] N. Saikia, "A parameter for Ramanujans function $\chi(q)$ : its explicit values and applications," ISRN Computational Mathematics, vol. 2012, Article ID 169050, 14 pages, 2012. 


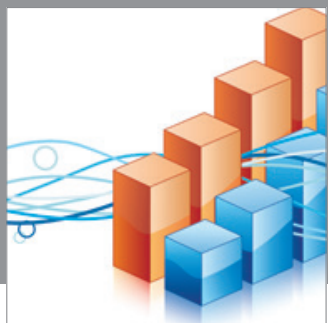

Advances in

Operations Research

mansans

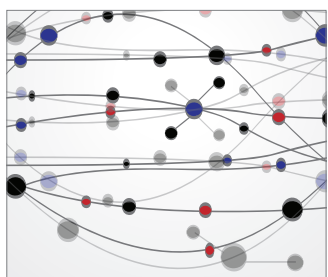

The Scientific World Journal
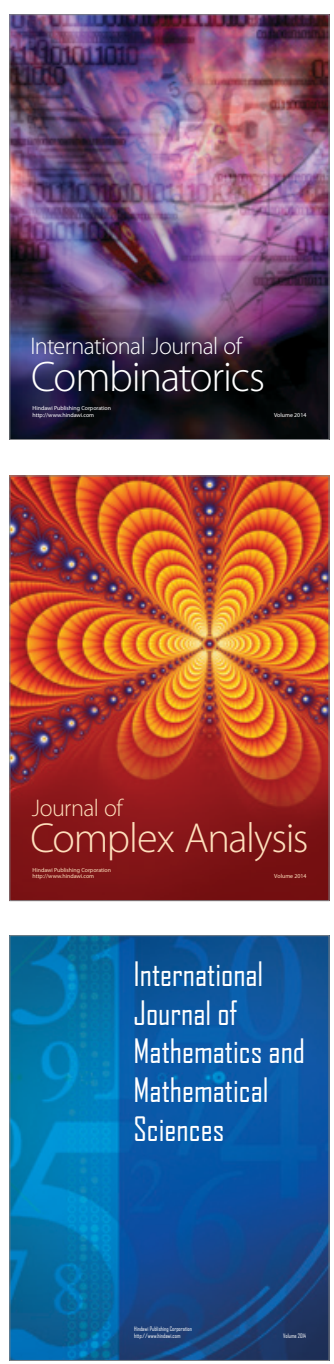
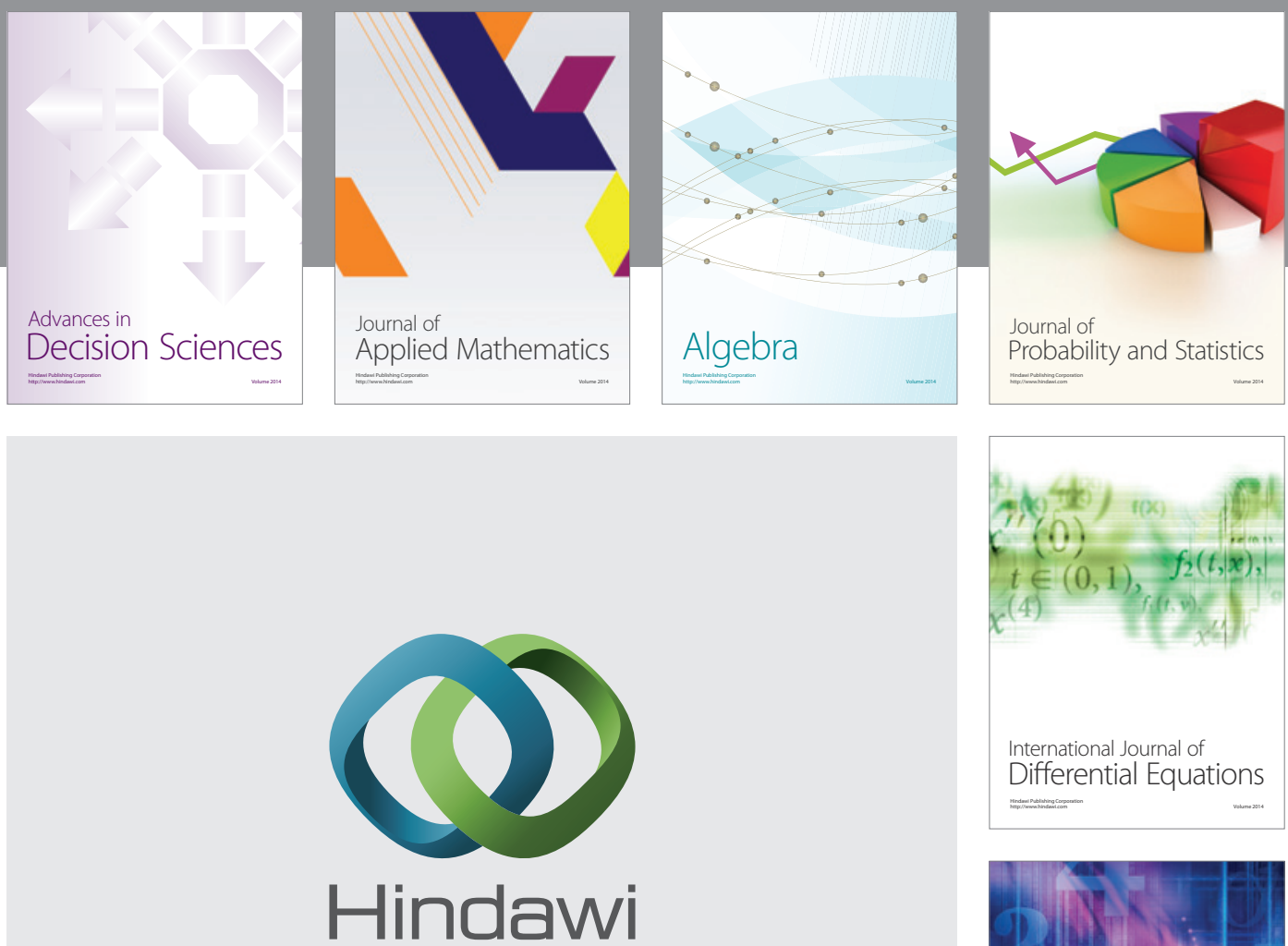

Submit your manuscripts at http://www.hindawi.com
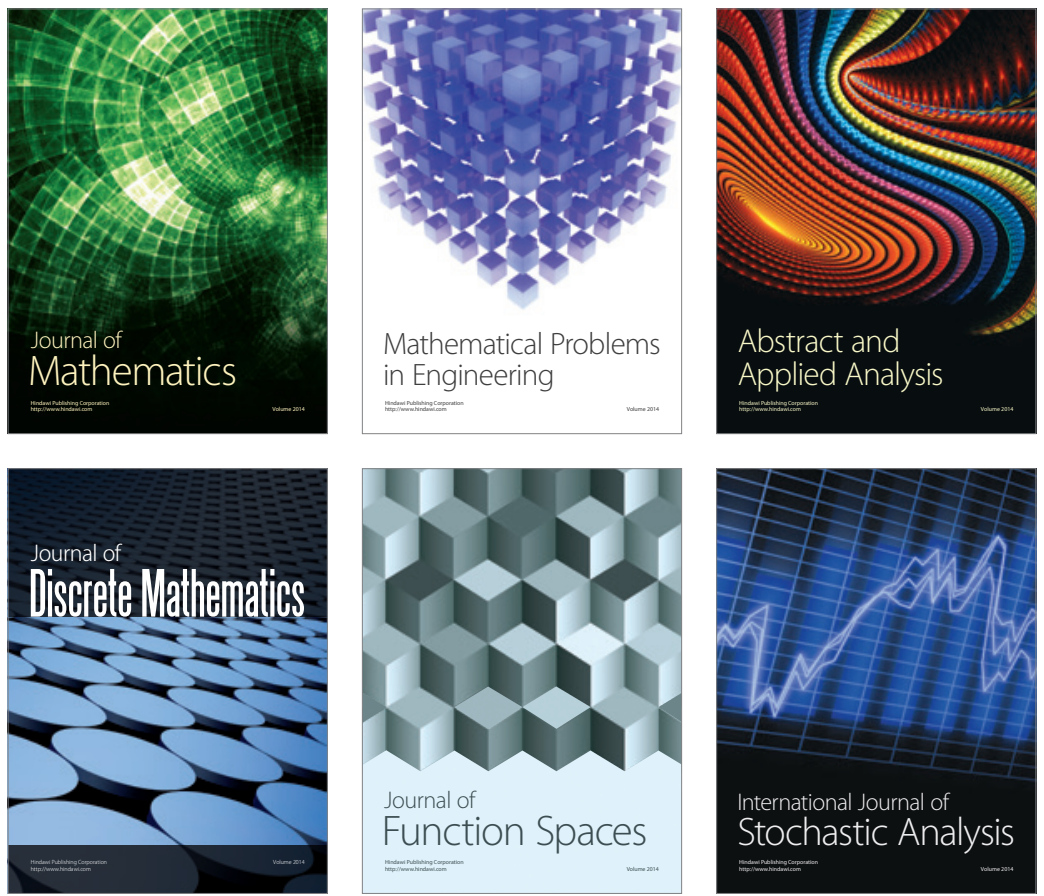

Journal of

Function Spaces

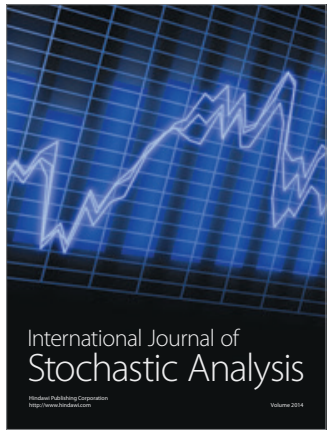

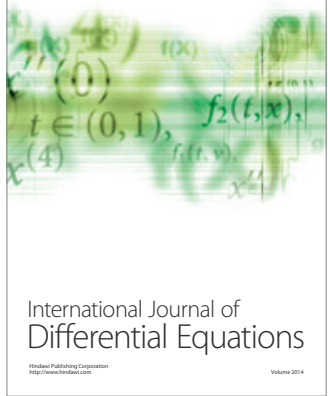
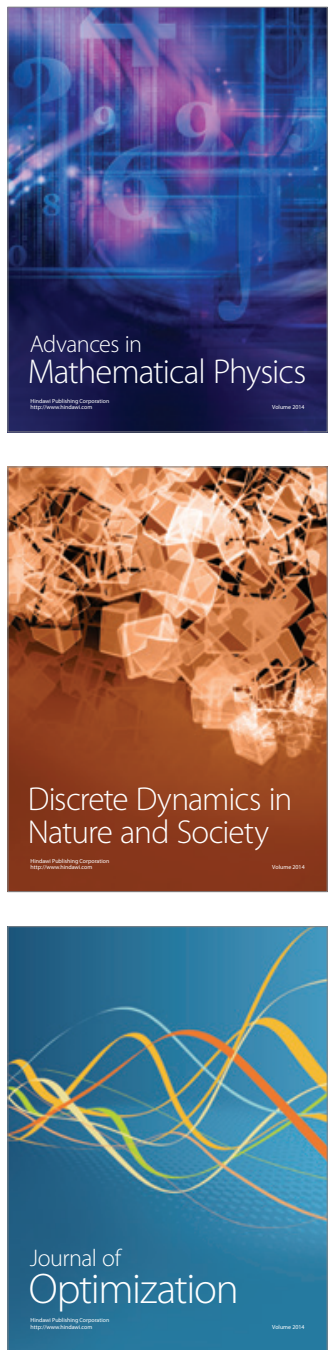